\title{
Ist pränatale Diagnostik diskriminierend?
}

\section{Intervention in eine Debatte}

\author{
Kirsten Achtelik
}

Journal für Psychologie, 26(2), 74-92

https://doi.org/10.30820/8248.05

www.journal-fuer-psychologie.de

\section{Zusammenfassung}

Pränatale Diagnostik gilt unter behindertenpolitischen und feministischen Aktivist*innen und Wissenschaftler*innen als ableistisch und diskriminierend. Dieses Argument findet in ethischen und politischen Debatten große Aufmerksamkeit, weil die Schlechterbehandlung von Minderheiten in demokratischen Gesellschaften als illegitim gilt. Der Diskriminierungsvorwurf wird allerdings auch als ungerechtfertigt abgelehnt, vor allem von Soziolog*innen und Ethiker*innen. Obwohl sich das Argument explizit ausschließlich gegen pränatale Diagnostik (PND) richtet, scheint der Schwangerschaftsabbruch nach einer solchen Diagnose häufig der eigentliche Auslöser für die Kritik zu sein. In diesem Text will ich das etwas unübersichtliche Diskursfeld beleuchten und den verschiedenen Argumenten nachgehen. Dazu ist eine Reflexion über einen für die Fragestellung passenden Diskriminierungsbegriff genauso nötig wie eine Präzisierung des verwendeten Modells von Behinderung. Die hier dargestellten Überlegungen eröffnen weiterführende Fragestellungen und sollen eine Grundlage für die Debatte um die diskriminierenden Effekte der PND schaffen.

Schlüsselwörter: Pränataldiagnostik, Behinderung, Diskriminierung, Ableism, soziales Modell

\section{Summary}

Is prenatal diagnostics discriminating against disabled people?

An intervention into a debate

Prenatal diagnosis is considered by disabled and feminist activists and scientists as ableist and discriminatory. This argument attracts a great deal of attention in ethical and political debates because the poorer treatment of minorities in democratic societies is considered illegitimate. However, the accusation of discrimination is also rejected as unjustified, especially by sociologists and ethicists. Although the argument is explicitly directed exclusively against prenatal diagnosis, abortion often seems to be the actual trigger for criticism after such a diagnosis. In this text I want to shed light on the somewhat confusing discourse and examine 
the various arguments. This requires a reflection on an adequate concept of discrimination as well as a clarification of the underlying model of disability. The considerations presented here open up further questions and a basis for the debate on the discriminatory effects of prenatal diagnosis.

Keywords: prenatal diagnosis, disability, discrimination, ableism, social model

\section{Problemaufriss}

Diskriminierung, verstanden als ungerechtfertigte Schlechterbehandlung marginalisierter Gruppen, gilt als inakzeptabel und ist mit dem Selbstverständnis moderner, westlicher Gesellschaften nicht unvereinbar. Daher gab und gibt es virulente gesellschaftliche Auseinandersetzungen darüber, was als Diskriminierung gelten kann und entsprechend zu bekämpfen ist.

Pränatale Diagnostik (PND), die nach möglichen Beeinträchtigungen des werdenden Kindes sucht, wird von vielen Kritiker*innen dieser Techniken als Diskriminierung von Menschen mit Behinderung ${ }^{1}$ verstanden. Einige Soziolog*innen lehnen diese These dagegen ausdrücklich ab. Dabei weist die Debatte jedoch die Eigentümlichkeit auf, dass die Beteiligten die Unterscheidung zwischen PND und Schwangerschaftsabbruch vernachlässigen bzw. nicht trennscharf durchhalten: Sie beantworten explizit nicht die Frage, ob und inwiefern PND diskriminierend wirkt, sondern die Frage, ob und inwiefern PND und sich daran anschließende Schwangerschaftsabbrüche eine Diskriminierung von Menschen mit Behinderung darstellen.

Ich halte es für eine produktive Diskussion über die diskriminierende Wirkung der PND für erforderlich, die pränatale Diagnostik analytisch von der eventuell darauf folgenden Abtreibung zu trennen. Zum einen ist die Debatte so komplex, dass es sinnvoll erscheint, zwei zwar aufeinander folgende und begründungslogisch verbundene Vorgänge getrennt zu untersuchen, zum anderen droht die moralisch stärker aufgeladene und anders konnotierte Debatte über (späte) Schwangerschaftsabbrüche (Achtelik, 2018) sonst die Reflexion über pränatale Diagnostik zu überschatten. Zudem beteiligen sich mit Feminist*innen und »Lebensschützern « Vertreter*innen zweier sozialer Bewegungen an der Debatte, die beide Reproduktionstechnologien kritisieren sowie mit dem Diskriminierungsargument arbeiten, in Bezug auf Schwangerschaftsabbrüche jedoch konträre Positionen vertreten: Die »Lebensschutz«-Bewegung will die Definition von Diskriminierung auf Embryonen und Föten ausdehnen, die feministischen und große Teile der behindertenpolitischen sozialen Bewegungen wollen die Auseinandersetzungen um ein Recht auf Abtreibung nicht durch die Problematisierung von Abbrüchen nach PND erschweren. 
Kritisiert wird in der Diskussion nicht jede pränatale Untersuchung, es wird vielmehr - wenn auch bisher unzureichend - versucht, medizinisch sinnvolle von potenziell diskriminierend wirkenden Untersuchungen zu unterscheiden. ${ }^{2}$ Als sinnvoll gelten Untersuchungen, deren Ergebnis therapeutische Möglichkeiten eröffnen, die sich positiv auf den Gesundheitszustand von werdender Mutter und werdendem Kind auswirken können. Als problematisch gelten solche, die nur nach einer potenziellen Abweichung oder Behinderung suchen und damit die Frage nach einem Abbruch nahelegen. ${ }^{3}$ Letztere habe ich als »selektive Pränataldiagnostik « (Achtelik, 2015) bezeichnet. Diesen Begriff werde ich in diesem Text allerdings nicht benutzen, da explizit die Diagnostik und der möglicherweise darauf folgende Abbruch analytisch getrennt werden sollen.

Diese kritische Sicht auf die pränatale Suche nach Normabweichungen und Hinweisen auf Behinderungen nimmt das in den Disability Studies entwickelte »soziale Modell « auf, das Behinderung nicht als ausschließlich körperlich-medizinisches Phänomen, sondern auch als Resultat gesellschaftlicher Herstellung und Problematisierung versteht. Zentral hierbei ist die Frage nach der Bedeutung, die Behinderung auf gesellschaftlicher, politischer und kultureller Ebene sowie für die Betroffenen hat. Das dominante negative Bild von Behinderung spielt sowohl für die gesellschaftliche Wahrnehmung von PND eine große Rolle als auch für die wissenschaftliche Bewertung der Frage, ob und warum PND als diskriminierend gelten kann.

Ich werde im Folgenden die soziologische Diskriminierungsforschung darstellen und nach einem für die Fragestellung geeigneten Diskriminierungsbegriff durchsehen. Sodann werde ich die bisherigen Argumente auf ihren Diskriminierungs- und Behinderungsbegriff sowie auf ihre Plausibilität prüfen, um im Anschluss die einzelnen Argumente daraufhin zu untersuchen, ob die behauptete Diskriminierung vorliegt. Im Resümee kläre ich, ob die Frage beantwortet werden kann, und zeige Vorschläge und Desiderate für die künftige Forschung auf.

\section{Soziologische Diskriminierungsforschung}

Dem lateinischen Wortstamm nach bedeutet $\gg$ Diskriminierung « zunächst nur Unterscheidung, also die Wabrnehmung von angenommenen oder tatsächlich vorhandenen Differenzen ohne eine Bewertung. Im Deutschen wird das Wort allerdings kaum in diesem neutralen, sondern hauptsächlich im negativen Sinne verwendet: Wird eine Handlung als diskriminierend bezeichnet, so schließt dies eine moralische Kritik an besagter Handlung bereits mit ein (Boshammer, 2008).4 In den aktuellen sozialwissenschaftlichen Debatten zur Diskriminierung besteht Einigkeit darüber, dass Diskriminierung kein gesellschaftlich überwundenes Problem, sondern ein aktuelles Phänomen ist (ebd.). Es wird allerdings eine Unschärfe des Diskriminierungsbegriffs beklagt, die 
sich Albert Scherr zufolge darin ausdrückt, dass eine »eigenständige, genuin soziologische Diskriminierungsforschung [...] bislang in deutschsprachigen Kontexten nicht etabliert « ist (Scherr, 2017, S. 45). . $^{5}$

Im Wörterbuch der Soziologie wird Diskriminierung als die »wahrgenommene ungerechtfertigte Schlechterbehandlung von Mitgliedern einer sozialen Gruppe oder einer sozialen Kategorie allein auf der Basis ihrer Gruppen- bzw. Kategoriemitgliedschaft « (Jonas, 2014, S. 81; H.i.O.) definiert. Das Phänomen wird mit dem Begriff der sozialen Diskriminierung als gesellschaftliches gefasst, um es »von individueller Schlechterbehandlung abzugrenzen«. Gleichzeitig sei der Begriff »subjektiv und wird aus einer Opferperspektive definiert « (ebd.). Um zu einem objektiven Konsens darüber zu kommen, welches Verhalten als diskriminierend zu gelten habe, müssten die »Perspektivendivergenzen «, also die unterschiedlichen Wahrnehmungen und Ansichten zwischen »Opfern, Tätern und nicht direkt betroffenen Gruppen ausgehandelt werden $\ll$ (ebd.).

Ob ein solcher objektiver Konsens möglich und wünschenswert ist, scheint allerdings fraglich, schließlich resultieren Diskriminierungen nicht aus Meinungsunterschieden, die durch Gespräche möglicherweise ausgeräumt werden könnten, sondern aus Machtdivergenzen. Da Diskriminierungen dazu beitragen, unterdrückte Gruppen in ihrer benachteiligten Position zu fixieren, tendieren Mitglieder unterdrückender Gruppen dazu, diskriminierendes Verhalten als unproblematisch und legitim wahrzunehmen und zu verteidigen, um so, bewusst oder unbewusst, ihre privilegierte Position zu verteidigen. Die Klärung der Frage, ob und inwiefern PND als Diskriminierung zu fassen ist, erfordert also auch eine Reflexion der gesellschaftlichen Macht- und Herrschaftsverhältnisse.

In dem soziologischen Sammelband Diskriminierung: Grundlagen und Forschungsergebnisse machen die Herausgeber*innen Ulrike Hormel und Albert Scherr (2010) eine Gemeinsamkeit der neueren sozialwissenschaftlichen Diskriminierungsforschung darin aus, dass diese sich nicht mehr » auf eine handlungstheoretische oder gruppenbezogene Analyse diskriminierender Einstellungen und Handlungen beschränkt, sondern auch gesellschaftsstrukturelle (ökonomische, politische, rechtliche), kulturelle (Diskurse und Ideologien), institutionelle sowie organisatorische Bedingungen und Formen von Diskriminierung in den Blick nimmt « (Hommel \& Scherr, 2010, S. 11). Dieser weite Diskriminierungsbegriff erlaube es, Unterscheidungen zwischen sozialen Gruppen zu fokussieren, »die mit Annahmen über spezifische Eigenschaften der >Angehörigen< der so unterschiedenen Gruppen einhergehen sowie mit sozialen und/oder ökonomischen und/oder politischen und/oder rechtlichen Benachteiligungen verschränkt sind « (ebd.). Für meine Fragestellung ist die explizite Frage nach »den sozialen Prozessen, in denen diskriminierende Unterscheidungen hervorgebracht werden sowie den sozialen Bedingungen, unter denen diese plausibel erscheinen « (ebd.) weiterführend. 
Im Handbuch Diskriminierung weisen Aladin El-Mafaalani, Julian Waleciak und Gerrit Weitzel (2017) in ihrem Beitrag zu tatsächlicher, messbarer und subjektiv wahrgenommener Diskriminierung darauf hin, dass $\gg$ sich die Ebenen der (objektiv) messbaren Teilhabechancen auf der einen und der subjektiv erlebten Diskriminierung auf der anderen Seite nicht ohne weiteres ineinander überführen « ließen (El-Mafaalani et al., 2017, S. 174). In international vergleichenden Studien ergab sich in Bezug auf Rassismus das unerwartete Bild, dass ein geringes Niveau von objektiver Diskriminierung mit einem hohen Niveau an wahrgenommener Diskriminierung korrespondiert, und umgekehrt (ebd., S. 175). Als Erklärung führen die Autor*innen unter anderem an, dass »der zentrale Referenzrahmen des Diskriminierungsbegriffs >Legitimität - Illegitimität $<\ll$ (ebd., S. 180) gesellschaftlich variabel ist, also je nach gesellschaftlicher Stellung, persönlicher Sensibilisierung und spezifischen Erfahrungsräumen sehr unterschiedlich ausfallen kann.

Aus einer medizinethisch-philosophischen Perspektive formuliert Andreas Kösters (2014) in seiner Dissertation Vorgeburtliche Selektion und Diskriminierung von Menschen mit Behinderungen zwei Kriterien, die vorliegen müssten, damit man von einer Diskriminierung ausgehen könne: »1. eine Benachteiligung von Individuen der betroffenen Gruppe und 2. die Ablehnung des der Diskriminierung zugrunde liegenden Merkmals als hinreichende Grundlage für diese Benachteiligung « (Kösters, 2014, S. 86). Beide Kriterien seien wichtig, da »unklar wird, warum es sich bei Diskriminierung um ein moralisches Problem handeln soll, wenn dadurch niemand (absolut oder relativ) schlechter gestellt wird « (ebd.) und »es in einigen Fällen moralisch durchaus akzeptiert ist, dass Menschen in bestimmten Situationen unterschiedlich behandelt werden, auch wenn dies für einige eine Benachteiligung bedeutet « (ebd., S. 87). AuBerdem weist er auf das Problem der mittelbaren Diskriminierung hin, die vorliegt, wenn » scheinbar neutrale Regeln, Strukturen etc. zu einer Benachteiligung bestimmter Gruppen führen « (ebd., S. 90).

Dass Menschen mit Behinderung gesellschaftlich auf vielen Ebenen diskriminiert werden, ist unstrittig. Alle Arten von Ungleichbehandlung aufgrund des Merkmals Behinderung als ungerechtfertigt und damit als diskriminierend zu verstehen, ist allerdings z. B. in Bezug auf die schulische Inklusion keineswegs allgemeiner Konsens (Füller, 2018). Das individualistisch-medizinische Modell von Behinderung, das diese als $\gg$ Problem, das behandelt, kuriert, therapiert oder rehabilitiert werden muss «, als »Abweichung vom normalen Gesundheitszustand « (Degener, 2015, S. 115) definiert, trägt dazu bei, dass die Beeinträchtigung als legitimer Grund für Ungleichbehandlung und gesellschaftlichen Ausschluss angesehen wird (ebd.). In den Disability Studies wird Behinderung dagegen vorrangig als soziales Phänomen verstanden, in dem gesellschaftliche Normen, strukturelle Gegebenheiten und körperliche Eigenschaften (Beeinträchtigungen) zusammenkommen. Diskriminierende Verhaltensweisen, Struk- 
turen und Diskurse gegen und über Menschen mit Behinderung werden als Ableism definiert. Ableism bezeichnet das diskriminierende Muster, Menschen anhand ihrer vermeintlichen Fähigkeiten (abilities) zu beurteilen und diese mit als normal geltenden körperlichen und geistigen Fähigkeiten abwertend zu vergleichen. Die Disability Studies-Professorin Swantje Köbsell zeigt, dass Ableism keine außergewöhnliche Ausnahme ist, sondern vielmehr ein die Gesellschaft »strukturierendes Verhältnis « (Köbsell, 2015, S. 21):

»Ableism durchzieht alle gesellschaftlichen Bereiche, wird in uns alle hineinsozialisiert, beeinflusst so die Einstellungen und Haltungen aller Mitglieder einer Gesellschaft und damit auch ihre Handlungen, die, wenn sie unreflektiert und unhinterfragt bleiben, [...] zur Konstruktion von Behinderung und Normalität beitragen « (ebd., S. 26).

Obwohl auch der Begriff der Behindertenfeindlichkeit diese Dimension beinhaltet, vor allem in der Verwendung durch Birgit Rommelspacher (1999), verleitet er doch zu unterkomplexen, personenzentrierten Analysen, weswegen ich den Ableism-Begriff verwende.

Ergänzend zu diesen grundsätzlichen Definitionsvorschlägen halte ich es für sinnvoll, die konzeptionellen Überlegungen zu »genetischer Diskriminierung « von Thomas Lemke (2006) einzubeziehen. Diese soll die Ungleichbehandlung von Menschen aufgrund vermuteter oder tatsächlich vorhandener genetischer Merkmale erfassen und wird meist ausdrücklich von der Diskriminierung Behinderter und (chronisch) Kranker abgegrenzt (ebd., S. 59). Im Bereich der pränatalen Diagnostik, die zum großen Teil auf genetischen Tests basiert, ergeben sich Schnittmengen zwischen den Diskriminierungsformen. Lemke kann zeigen, dass diese angenommene Unterscheidung zwischen genetischer Diskriminierung und Diskriminierung aufgrund von Behinderung empirisch kaum durchzuhalten (ebd., S. 67ff.) und für die Bekämpfung ungerechtfertigter Diskriminierung nicht sinnvoll ist (ebd., S. 72ff.).

Er kritisiert die » begrifflichen Unschärfen « bisheriger Konzepte und schlägt einen erweiterten Begriff vor, der organisationale, interaktionelle und institutionelle Diskriminierung umfasst (Lemke, 2010, S. 324). Organisationale Diskriminierung bezeichnet die von Organisationen wie Krankenkassen oder Behörden ausgehende Benachteiligung, Missachtung und Ausgrenzung und ist bisher am besten empirisch untersucht worden. In die interaktionelle Diskriminierung, die zwischen Menschen stattfindet, bezieht Lemke explizit auch »Missachtung und Stigmatisierung « (ebd., S. 329) ein. Institutionelle Diskriminierung zielt auf die Ebene der »Diskurse und Praktiken [...], die mittelbar auf Betroffene einwirken und deren Entscheidungsspielräume und Handlungsoptionen beschränken «. Um diese Art der Diskriminierung in den Blick zu nehmen, sei es notwendig, » auch jene sozio-kulturellen Regeln zu analysieren, die an 
alle Gesellschaftsmitglieder adressiert sind «, die »normativen Handlungsmuster und kulturellen Selbstverständlichkeiten, die Unwerturteile, Vorurteilsstrukturen und Formen von Missachtung (re-)produzieren und prägen « (ebd., S. 335). Mit einem diese drei Ebenen umfassenden Diskriminierungsbegriff können die Wechselwirkungen zwischen strukturellen Vorgaben (Krankenkassenfinanzierung, gesetzlichen Regelungen), persönlichen Entscheidungen und gesellschaftlichen Normen (Ableism) wahrgenommen und analysiert werden. Das Konzept der »indirekten Diskriminierung « umfasst alle Faktoren, die »mittelbar auf Betroffene einwirken und deren Entscheidungsspielräume und Handlungsoptionen beschränken « (Lemke, 2006, S. 63), und durchzieht die drei genannten Ebenen.

Ein weiter Diskriminierungsbegriff, der die verschiedenen Ebenen und indirekten Prozesse fassen kann, ist notwendig, um die mögliche diskriminierende Wirkung pränataler Diagnostik analysieren zu können. Mit dem Begriff des Ableism können Diskriminierungen nicht nur als beklagenswerte Ausnahmen, sondern als die Normalität strukturierende Handlungen, Einstellungen und Deutungsmuster verstanden werden, die deswegen jedoch nicht weniger problematisch sind.

\section{Die Debatte um die Wirkung von PND}

$\mathrm{Ob}$, wie und gegen wen pränatale Diagnostik diskriminierend wirke, wird politisch und ethisch diskutiert. Dabei werden verschiedene Argumente und Gegenargumente entwickelt, die ich im Folgenden anhand exemplarischer Texte näher beleuchte.

Das Expressivist Argument wurde in den angloamerikanischen Disability Studies Ende der 1980er Jahre entwickelt - einen Überblick über dessen Verwendung geben Erik Parens und Adrienne Asch (2000). Ihnen zufolge resultiert Diskriminierung für die Vertreter*innen von Behindertenrechten in den USA dann, » when people in one group fail to imagine that people in some $>$ other $<$ group lead lives as rich and complex as their own « (ebd., S. 8). Dieser Fehler, nämlich anzunehmen, Menschen einer anderen Gruppe, in diesem Fall Behinderte, könnten gar kein Leben führen, das so reichhaltig und komplex wie ihr eigenes sei, motiviere auch werdende Eltern zur Vermeidung von Kindern mit Behinderung. Die zentrale Annahme des Expressivist Argument lautet, dass eine bestimmte Eigenschaft des werdenden Kindes - eben die Behinderung - alle anderen überdecke und ausreiche, um sich ein qualitativ hochwertiges Leben für das zukünftige Kind und ein Leben mit ihm nicht mehr vorstellen zu können (ebd., S.13). Durch die Abtreibung des behinderten Fötus werde diese Lebensunwertannahme ausgedrückt, und zwar durch die Handlung selbst. Es kommt also bei diesem Argument nicht auf mögliche Folgen an. Dies sei für Menschen mit Behinderung verletzend, da das Reduziertwerden auf die Behinderung ihrer täglichen Erfahrung entspreche (ebd., 
S. 14). Einig sind sich die Autor*innen, die dieses Argument nutzen, darin, dass weder der Schwangerschaftsabbruch an sich kritisiert werden soll noch die ehemals schwangere Frau (ebd., S. 12). Allerdings ist die Tendenz zu beobachten, sich mit dem Fetus zu identifizieren und das werdende Kind mit Behinderung bereits vor der Geburt in die Community der Menschen mit Behinderung aufzunehmen (ebd., S. 14).

Das Argument wird häufig verwendet, aber selten präzisiert. So bleibt unklar, weshalb die Diagnostik bereits als diskriminierend angesehen wird, wenn das Argument nicht ohne den Bezug auf den nachfolgenden Schwangerschaftsabbruch funktioniert, und wieso die ehemals Schwangere von dem Diskriminierungsvorwurf ausgenommen werden kann, wenn es doch ihre Entscheidung ist, die das angebliche Unwerturteil ausdrückt. Dieter Birnbacher (2006, S. 329) problematisiert in seiner Diskussion dieses Argumentes die Annahme eines bestimmten Urteils ohne Intention der vermeintlich urteilenden Person. Sibylle Volz weist in ihrer an das Expressivist Argument anschließenden Untersuchung des Diskriminierungsgehalts von PND die Notwendigkeit einer $\gg$ bewusste[n] diskriminierende[n] Absicht « allerdings zurück (Volz, 2003, S. 74). Dennoch scheint der Name Expressivist Argument nicht gut gewählt, wenn es nicht so sehr darum geht, was durch eine Handlung ausgedrückt wird oder werden soll, sondern darum, wie sie aufgenommen wird.

Die UN-Behindertenrechtskonvention (UN-BRK) ist ein wichtiges Instrument zur gesetzlichen und sozialen Gleichstellung von Menschen mit Behinderung. Die Konvention basiert auf einer weiten Diskriminierungsdefinition: Nach Artikel 2 umfasst der Begriff

»jede Unterscheidung, Ausschließung oder Beschränkung aufgrund von Behinderung, die zum Ziel oder zur Folge hat, dass das auf die Gleichberechtigung mit anderen gegründete Anerkennen, Genießen oder Ausüben aller Menschenrechte und Grundfreiheiten im politischen, wirtschaftlichen, sozialen, kulturellen, bürgerlichen oder jedem anderen Bereich beeinträchtigt oder vereitelt wird « (United Nations, 2006, S. 4).

Dieser verpflichtet mit Artikel 8 die Vertragsstaaten und ihre untergeordneten Instanzen dazu, »Klischees, Vorurteile und schädliche Praktiken gegenüber Menschen mit Behinderungen [...] in allen Lebensbereichen zu bekämpfen « (ebd., S. 9). Versuche, mit der UN-BRK gegen pränatale Untersuchungen zu argumentieren, stützen sich hauptsächlich auf diesen Artikel und sein positives Ziel der Bewusstseinsbildung. Neben einigen zivilgesellschaftlichen und behindertenpolitischen Gruppen vertritt vor allem die Humangenetikerin und Sozialethikerin Sigrid Graumann die Ansicht, dass PND und Präimplantationsdiagnostik »in dem hier gemeinten Sinn schädliche Praktiken gegenüber Menschen mit Behinderung darstellen und aus diesem Grund nicht mit dem Geist der UN-BRK vereinbar « seien (Graumann, 2014, S. 73). 
Kösters (2014, S. 3f.) kommt in seiner philosophisch angelegten Prüfung der Diskriminierungsvorwürfe und ihrer oft impliziten Vorannahmen auf Kohärenz und Plausibilität zu dem Schluss, dass keines der Argumente stark genug sei, Verbotsforderungen mit einem Verstoß gegen das Diskriminierungsverbot zu begründen (ebd., S. 221). Die Philosophin Weyma Lübbe (2003, S. 204) prüft, ob es sich bei der Ungleichbehandlung behinderter Föten um ungerechtfertigte Ungleichbehandlung handelt. Dies wäre dann der Fall, wenn die von den werdenden Eltern angenommene Mehrbelastung durch ein Kind mit Behinderung unzutreffend wäre - ob diese eintrete oder nicht, sei jedoch individuell verschieden. Die Anwendung des im Grundgesetz verankerten Diskriminierungsverbotes auf persönliche Nahbeziehungen wie die zwischen werdender Mutter und werdendem Kind lehnt sie zudem als unangemessen ab (ebd., S. 215).

Der Jurist und Soziologe Wolfgang van den Daele untersucht in seinem vielzitierten Text »Vorgeburtliche Selektion: Ist die Pränataldiagnostik behindertenfeindlich? « anhand »empirische[r] Indikatoren für steigende Diskriminierung « (van den Daele, 2005, S. 99) den »Realitätsgehalt « (ebd.) des Diskriminierungsvorwurfs. Van den Daele kann zeigen, dass Menschen mit Behinderung in Bezug auf die von ihm gewählten Indikatoren (»Abbau von Rechtspositionen «, »Rücknahme sozialpolitischer Leistungen «, »Zunahme ablehnender Einstellungen «) (ebd., S. 100) keiner zunehmenden Diskriminierung ausgesetzt sind. Die subjektive Besorgnis von behinderten Menschen, die sich »als Person infrage gestellt und als unerwünscht abgestempelt sehen, wenn ungeborene Kinder abgetrieben werden, weil sie ebenso behindert sein würden, wie sie selber «, findet der Soziologe zwar » nachvollziehbar «, dies sei jedoch nicht als Diskriminierung zu fassen, da es nicht der »Intention derjenigen, die vorgeburtliche Selektion praktizieren «, entspräche (ebd., S. 115). Er schlägt dafür den Begriff der »Kränkung (Stigmatisierung) « (ebd., S. 119) vor, ohne jedoch zu begründen, warum für eine Diskriminierungshandlung zwingend eine Absicht vorliegen müsse. Der Autor arbeitet mit einem engen Begriff von Behindertenfeindlichkeit, er interpretiert PND nur als Ausdruck einer legitimen Ablehnung von Behinderung und nicht einer illegitimen Ablehnung von Menschen mit Behinderung. Die sich im »Defizitmodell « (ebd., S. 115) niederschlagende Deutung der Behinderung als Verlust hält er aus der Perspektive nicht behinderter Menschen für normal und nachvollziehbar, die Problematisierung dieser Denkweise vonseiten »manche[r] Behindertenverbände « und der Disability Studies lehnt er als »forciert « (ebd., S. 117) ab. Die in seinen Augen legitimen Wahrnehmungsunterschiede dürften nicht dazu führen, »die große Mehrheit der Bevölkerung als behindertenfeindlich einzustufen « (ebd., S. 109).

Auch der Philosoph Andreas Kuhlmann versteht die »Bereitschaft zur Selektion « (2011, S. 97), die sich in den Tests ausdrücke, als zumindest potenziell verletzend für Menschen mit Behinderung. Diesen würde so eine »mindere Wertschätzung signalisiert «, außerdem würde der »demokratische Konsens, dass Minderheiten nicht 
diskriminiert [...] werden sollen, [...] in Frage gestellt « (ebd., S.98). Das ist eine weitreichende Schlussfolgerung, zu der der selbst behinderte Kuhlmann allerdings nur kommen konnte, weil er davon ausging, dass die Tests meistens mit dem Ziel vorgenommen würden, bei einem Befund die Schwangerschaft abzubrechen. Dies läuft aber seiner eigenen Charakterisierung der Tests als routinemäßig durchgeführte, also kein spezifisches Ziel verfolgende Untersuchungen zuwider. Er wendet sich zudem gegen das soziale Modell von Behinderung, indem er dessen Vertreter*innen unterstellt, sie würden jegliche reale körperliche Einschränkung negieren.

Die Soziologin und Humanmedizinerin Tanja Krones lehnt, auf Einstellungsuntersuchungen und Expert*inneninterviews der Arbeitsgruppe Bioethik-Klinische Ethik am Zentrum für Konfliktforschung in Marburg über PID und PND gestützt, sowohl das Expressivist Argument als auch Dammbruchszenarien ab: Zu ambivalent seien die Befragten, sowohl in ihren Einstellungen gegenüber behinderten Menschen als auch in Bezug auf ihr Konzept von Behinderung generell, um von ihren Aussagen einen Diskriminierungsvorwurf als erhärtet anzusehen (Krones, 2008). Einstellungsuntersuchungen sind jedoch gerade bei tabuisierten Themen vorsichtig zu bewerten. Wie ein Forschungsprojekt zu Diskriminierung im Alltag zeigt, sind Ambivalenzen im Verhältnis von Menschen mit und ohne Behinderung zueinander eher der Normalzustand als die Ausnahme. Sie können daher nicht als Beleg angeführt werden, dass keine Behindertenfeindlichkeit vorliege, wie Krones das tut. Behindertenfeindlichkeit komme sehr häufig in Ekelgefühlen und dem Bedürfnis, sich »diese Menschen, in der wörtlichen Bedeutung, möglichst vom Leibe [zu] halten « (Flaig, 2009, S. 73), zum Ausdruck, werde aber als moralisch verwerflich empfunden - daher die Schuldgefühle und die Ambivalenzen.

In der Debatte wird häufig die Vermutung vorgebracht, dass sich die Diskriminierung von behinderten Menschen durch die Anwendung der Techniken verstärkt habe oder verstärken könnte (Graumann, 2014, S. 80; Volz, 2003, S. 84). Dies ist jedoch, wie Kösters (2014, S. 185) zeigt, nur schwierig nachweisbar, da die vorhandenen statistischen Erhebungen keine Entwicklungen abbilden und solche Trends sich nicht kausal auf die Pränataldiagnostik zurückführen ließen. Solche Befürchtungen und SlipperySlope-Argumente können also weder verifiziert noch falsifiziert werden.

Festzuhalten ist, dass keine ${ }^{*} \mathrm{r}$ der Autor*innen eine analytische Trennung vollzieht zwischen der möglichen diskriminierenden Wirkung der Untersuchungstechniken und der möglichen diskriminierenden Wirkung der danach erfolgenden Schwangerschaftsabbrüche. Insofern ist die These, dass pränatale Diagnostik als solche diskriminierend sei, noch gar nicht überprüft worden. Unklar bleibt bei den Befürworter*innen wie auch bei den Gegner*innen der Diskriminierungsthese, was genau unter Diskriminierung verstanden wird, meist fehlt eine explizite Definition. Daher bleibt vieles vage und bei der Durchsicht der Standpunkte entsteht der Eindruck, dass in der Debat- 
te hauptsächlich aneinander vorbeigeredet wird (ebd., S. 224). Weder überzeugt das Expressivist Argument, da unklar bleibt, wie und wodurch das angeblich mit PND verbundene Unwerturteil ausgedrückt werde, noch scheint eine nicht erfolgte positive Bewusstseinsbildung als menschenrechtsverletzende Diskriminierung plausibel. Allerdings haben weder die Einstellungsabfragen noch die sozioökonomischen Daten über die Lebensqualität behinderter Menschen den Diskriminierungsvorwurf widerlegen können.

\section{Diskussion der Argumente: Ist PND diskriminierend?}

Ich werde im Folgenden Lemkes Konzept von interaktioneller, organisationaler und institutioneller Diskriminierung auf verschiedene Topoi und Studienergebnisse über pränatale Diagnostik anwenden. Zur zwischen Menschen stattfindenden, interaktionellen Diskriminierung zählen Beleidigungen und Stigmatisierungen, die aufgrund des Merkmals »Behinderung « erfolgen. Die formalen Regeln und Routinen, die sich in Organisationen wie Krankenkassen und Arztpraxen herausgebildet haben, können eine organisationale Diskriminierung der Betroffenen bewirken. Kulturelle Normen und Diskurse wie die mit dem medizinischen Modell verbundene Abwertung von Behinderung und die Verknüpfung von Beeinträchtigungen mit Leiden lassen sich unter institutionelle Diskriminierung fassen. Die drei Diskriminierungsformen können Wechselwirkungen miteinander eingehen und sich gegenseitig verstärken. Den Schwerpunkt der Analyse werde ich auf die institutionelle Diskriminierung legen, entsprechend des Ansatzes der Disability Studies.

Die Frage an Eltern von Kindern mit Behinderung, ob sie von der Behinderung nicht vorher gewusst hätten, wird von Betroffenen und in der Literatur häufig erwähnt. $\mathrm{Zu}$ der realen Häufigkeit sowie zur $\mathrm{Zu}$ - oder Abnahme solcher Bemerkungen gibt es allerdings kaum Untersuchungen. ${ }^{6}$ Gerade Eltern von Kindern mit Trisomie 21 scheinen dem ausgesetzt zu sein, was auf einen Zusammenhang zwischen solchen Fragen und dem allgemein verbreiteten Wissen über eine Behinderung und ihre pränatale Diagnostizierbarkeit hinweist. Solche Fragen suggerieren sehr deutlich, dass ein Schwangerschaftsabbruch für wünschenswert gehalten worden wäre, wenn die Behinderung pränatal bekannt war. Dies stellt sozusagen den zweiten, unausgesprochenen Teil der Frage dar (wenn ihr von der Behinderung gewusst habt, warum habt ihr das Kind dann bekommen?). Solche Fragen sind sehr verletzend, sagen sie doch, dass das Kind, so wie es ist, besser nicht da wäre. Sie sind daher als interaktionelle Diskriminierung zu fassen, die sich aufgrund der Möglichkeiten pränataler Diagnostik und des verbreiteten Wissens darum gegen die Eltern behinderter Kinder und gegen die behinderten Kinder richtet. Pränatale Diagnostik trägt zwar zu dieser Art von Diskriminierung bei, 
da solche Aussagen nicht möglich wären bzw. keinen Sinn machen würden ohne die Möglichkeit, bestimmte Behinderungen pränatal zu erkennen. Da die Frage nur oberflächlich auf die Diagnostik zielt, sie aber eigentlich den Abbruch meint, kann dieser Komplex allerdings nicht als Beleg dafür herangezogen werden, dass PND an sich diskriminierend wirkt.

Die befürchtete Verschlechterung der gesellschaftlichen Stellung behinderter Menschen aufgrund der Pränataldiagnostik ist ein verbreiteter Topos. Laut einer Untersuchung von Lenhard, Henn, Ebert, Schindelhauer-Deutscher und Breitenbach (2005, S. 36) befürchteten $47,7 \%$ der Eltern von Kindern mit Trisomie 21 eine Verschlechterung der gesellschaftlichen Stellung behinderter Menschen aufgrund der Pränataldiagnostik. Was befürchtet wird und wie die befürchteten Verschlechterungen mit PND zusammenhängen sollen, bleibt meist vage, sodass der Topos nicht eindeutig einer Diskriminierungsebene zugeordnet werden kann. Auch dieses Loss of Support-Argument (vgl. Kösters, 2014, S. 184ff.) funktioniert wahrscheinlich nur in Verbindung mit dem Gedanken, dass mehr Diagnostik zu mehr Abbrüchen führt und zu weniger Menschen mit einer bestimmten Behinderung. Dies wiederum wird mit negativen Auswirkungen auf die gesellschaftlichen Einflussmöglichkeiten dieser Gruppe in Verbindung gebracht. Bisher sind solche Tendenzen, trotz jahrzehntelanger Ausweitung der Diagnostik, allerdings nicht zu beobachten. Auch aus Ländern, in denen der nicht invasive Bluttest (NIPT) flächendeckend angeboten wird und tatsächlich eine Abnahme der Geburtenzahl von Kindern mit Trisomie 21 zu beobachten ist, wie in Dänemark oder Island (Lou et al., 2018), wurde noch keine Verschlechterung des gesellschaftlichen Standings dieser Gruppe berichtet. Allerdings bilden die Familien aufgrund der Häufigkeit dieser Behinderung eine relativ große Community, die dadurch viele Ressourcen mobilisieren kann. Wenn diese Community schrumpft, hätte das vermutlich tatsächlich spürbare unangenehme Folgen für die verbliebenen Eltern. Dies als Diskriminierung zu werten, scheint mir den Begriff allerdings unzulässig auszudehnen. Zudem ist auch in diesem Fall nicht die Diagnostik das Problem, sondern die davon motivierten Schwangerschaftsabbrüche. Wenn durch andere Methoden die Zahl der Menschen mit einer (bestimmten) Behinderung sinken würde, etwa durch Impfungen oder durch das Verhüten schwerer Arbeits- oder Verkehrsunfälle, klänge die Warnung, solche Maßnahmen würden die gesellschaftliche Stellung behinderter Menschen verschlechtern, eher absurd. Andersherum kann der Abbau sozialer und gesundheitlicher Sicherungssysteme die Angst vor einem Kind mit Behinderung und damit den Wunsch nach pränataler Diagnostik ansteigen lassen.

Die Formulierung, Menschen mit Behinderung fühlten sich durch PND diskriminiert, wird häufig als Argument gegen pränatale Diagnostik ohne therapeutische Implikationen angeführt. Dies wird unter anderem darauf zurückgeführt, dass Menschen mit einer bestimmten Behinderung, die pränatal erkennbar ist, sich fragen müssten, was ih- 
re eigenen Eltern getan hätten (Birnbacher, 2006, S. 330). Dies kann jedoch nur für die Gruppe bereits erwachsener Behinderter gelten, deren Geburt vor der Entwicklung und Durchsetzung bestimmter Techniken liegt - die anderen müssten sich ja umso geliebter und angenommener fühlen, da ihre Eltern diese nicht angewandt oder daraus keine negativen Schlussfolgerungen gezogen haben. Häufiger wird das sogenannte KränkungsArgument in dem allgemeineren Sinne benutzt, dass durch die Bereitstellung von pränataler Diagnostik ohne therapeutischen Mehrwert ein gesellschaftliches Unwerturteil über damit diagnostizierbare Behinderungen und damit über die Menschen getroffen würde, die mit diesen Behinderungen leben. Das Gefühl, dass jemand ein Unwerturteil über jemand anderen abgibt, ist jedoch noch abstrakter und unklarer (v.a. in Bezug darauf, wie sich das überhaupt anfühlen könnte) als die Wahrnehmung, sich diskriminiert zu fühlen. Die Formulierung, sich »diskriminiert zu fühlen «, ist mittlerweile in die Umgangssprache eingegangen, es gibt jedoch meines Wissens nach keine Forschung zu der Verwendung dieses Ausdrucks oder dazu, wodurch »sich diskriminiert fühlen « von »sich ungerecht behandelt fühlen « unterscheidet. Ungerecht behandelt wird die Person mit Behinderung jedoch nicht, weil eine ihr unbekannte Schwangere eine bestimmte pränatale Untersuchung durchführen lässt. Auch die Ebene, auf der die Diskriminierung stattfinden würde, ist nicht eindeutig zu bestimmen. Bei einem derart komplexen Vorgang läge wohl v.a. institutionelle Diskriminierung vor, mit Anteilen auf den anderen Ebenen. Die kritische Debatte über pränatale Diagnostik hat diesen Topos eventuell erst geschaffen, ihn aber auf jeden Fall immens verstärkt. Mit Bezug auf El-Mafaalani et al. (2017) sind hier starke Rückkopplungseffekte der gesellschaftlichen Problematisierung von und Bewusstseinsbildung über PND zu erwarten - Kampagnen oder Diskurse, die PND-kritisch sind und negative Bilder von Behinderung aufbrechen wollen, könnten überhaupt erst dazu führen, dass Menschen mit Behinderung von diesem Problem erfahren, bzw. dazu, dass es ihnen stärker bewusst wird. Dies ist darum wahrscheinlich, weil sowohl pränatale Diagnostik, die über »Babyfernsehen « hinausgeht, als auch Schwangerschaftsabbrüche hochtabuisierte Themen sind, die normalerweise im privaten Bereich verbleiben und selten öffentlich thematisiert werden.

Volkswirtschaftliche Berechnungen zu möglichen Kostenersparnissen im Gesundheitswesen durch ein vermehrtes Angebot pränataler Diagnostik sind in Deutschland zu Recht verpönt. In den Anfangsjahren der Übernahme der Untersuchungskosten durch die Krankenkassen wurde das Kostenargument noch deutlich benannt. Dabei wurden die Kosten der Untersuchung (z. B. aller Schwangeren ab einem bestimmten Alter) mit den angenommenen Kosten für die Versorgung der geborenen Kinder mit Trisomie 21 aufgerechnet (Enquete, 2002, S. 71). Solche Berechnungen stellen einen klaren Fall von mittelbarer, organisationaler Diskriminierung dar. Sie in der Debatte als mögliche, befürchtete Folgen der weiteren Normalisierung und Liberalisierung der pränatalen Diagnostik aufzurufen, ist allerdings argumentativ nicht ganz sauber. Die 
Tabuisierung solcher Kosten-Nutzen-Berechnungen stellt vielmehr einen der großen diskursiven Erfolge der Behindertenbewegung dar, die zeitlich parallel zur Etablierung der PND entstand (Tolmein, 1990). Es ist daher kaum anzunehmen, dass die weitere Normalisierung von PND gegenteilige Auswirkungen habe. Eher könnten eine Stärkung antiemanzipatorischer gesellschaftlicher Tendenzen und starke Einschnitte im Gesundheitssystem zur Rückkehr der Kosten-Nutzen-Rechnung führen. Zudem funktionieren solche Berechnungen nur aufgrund der Annahme, dass eine große Mehrheit der Schwangeren, die ein positives Ergebnis erhalten, sich für einen Abbruch entscheiden würde. Würden die meisten die Tests nur nutzen, um sich besser auf die Geburt und das Leben mit ihrem Kind vorzubereiten, würden die Kosten für das Gesundheitssystem eher steigen bzw. wären gar nicht berechenbar. Auch diese Überlegungen funktionieren also nicht alleine in Bezug auf PND, sondern nur unter Miteinbeziehung der Abbruchsentscheidung.

Der in der Pränataldiagnostik vorherrschende medizinische Blick auf Behinderung kann nach Lemke (2010) als institutionelle und organisationale Diskriminierung von Menschen mit Behinderung verstanden werden. Die pränatale Suche nach nicht therapierbaren Normabweichungen wird, z. B. über Ultraschalluntersuchungen, als normalisierter Teil der Schwangerschaftsversorgung wahrgenommen. Dem liegt die normative Annahme zugrunde, dass Behinderungen sehr belastend und mit unnötigem Leiden verbunden sind. Diese Defizitorientierung der Untersuchungen erhält das ableistische, medizinische Modell aufrecht und verstärkt es, wohingegen das soziale Modell von Behinderung eine Reflexion der gesellschaftlichen Bedingungen ermöglichen würde. Die Vermeidung von Kindern mit Behinderung gilt dabei (u. a. wegen der »Euthanasie «-Verbrechen im Nationalsozialismus und der Erfolge der Behindertenbewegung; vgl. Weingart et al., 1992, S. 680f.) nicht mehr als legitimes Ziel. Vielmehr stehen die Untersuchungen unter dem Paradigma der reproduktiven Rechte von Frauen und deren legitimen Bedürfnis, Wissen über ihr zukünftiges Kind zu erlangen (Baldus, 2006, S. 24). Gleichzeitig gilt es als Zeichen der Verantwortungsübernahme für das werdende Kind, wenn sich die Schwangere vergewissert hat, dass keine Behinderung vorliegt. Diese gesellschaftliche Erwartungshaltung beschränkt die Handlungsoptionen der Schwangeren, weil eine Nichtteilnahme begründungsbedürftig wird, sowohl im Freundes-, Familien- und Bekanntenkreis als auch gegenüber der*/dem* jeweiligen Ärztin*/Arzt*(ebd., S. 185). An der Praxis des pränatalen Untersuchungsangebotes lässt sich zeigen, dass »auch formal freiwillige Handlungsoptionen und symmetrische Entscheidungssituationen «, wie sie in der Schwangerenversorgung der Fall sind, »diskriminierende Effekte haben können « (Lemke, 2010, S. 336). Die ableistischen Normen auf der institutionellen Ebene beeinflussen die Normalisierungspraktiken in Arztpraxen und Krankenkassen auf der organisationalen Ebene und beide die interaktionellen Erwartungshaltungen und Sagbarkeiten. 
Durch diese Analyse kommen schwangere Frauen und werdende Eltern von behinderten Kindern als potenziell von Diskriminierung durch pränatale Diagnostik betroffene Gruppen in den Blick. Konkret benennt Lemke die auch in den Debatten um pränatale Diagnostik viel diskutierten wachsenden Ängste (von Schwangeren vor einem Kind mit Behinderung) als relevante Faktoren: »[S] elbst wenn die Ängste übertrieben bzw. unrealistisch erscheinen mögen, haben sie doch konkrete individuelle und kollektive Folgen « (ebd., S. 338). In mehreren Studien wird der Aussage, durch die Tests sei diese Angst verstärkt bzw. erst ausgelöst worden, zugestimmt (Renner, 2006, S. 41). ${ }^{7}$ Nicht nur Menschen mit Behinderung, sondern auch Schwangere und werdende Eltern als von Diskriminierung durch PND bedrohte Gruppen zu deuten, kann den in dem Diskriminierungsvorwurf doch immer mitschwingenden Vorwurf gegen diese Gruppen und die implizit mitverhandelte Täter-Opfer-Dichotomie auflösen.

Eine Gruppe als von Diskriminierung bedroht zu deuten, die sich scheinbar zufrieden an der Aufrechterhaltung des Status quo beteiligt, scheint auf den ersten Blick überzogen. Die repräsentative Studie von Ilona Renner zeigt, dass 77\% der befragten Frauen im Nachhinein über die Untersuchungen froh waren und 69\% in einer erneuten Schwangerschaft wieder so handeln würden. Lediglich eine kleine Minderheit von knapp 4\% der Schwangeren findet, dass die PND $\gg$ zu starken Stress und Verunsicherung « (ebd., S. 42) bedeutete. Aber auch für das Leben von Menschen ohne Behinderung kann gesellschaftlicher Ableism eine (negative) Rolle spielen, für sie äuBert er sich hauptsächlich in ihrer eigenen Angst vor Behinderung. Anne Waldschmidt hat in Anschluss an Jürgen Link herausgearbeitet, dass die Denormalisierungsangst ein zentrales Moment im Diskurs und im Nachdenken der (noch) nicht Behinderten über behinderte Menschen ist. Zentrale Topoi sind die Angst vor Alter und Pflegebedürftigkeit sowie die Befürchtung, mit einem behinderten Kind selbst ins soziale Abseits zu geraten (Waldschmidt, 2009, S. 328). ${ }^{8}$ Diese Angst der $\gg$ Normalen « davor, fortan zu »den Behinderten « zu gehören, ist Teil des mit pränataler Diagnostik verbundenen Ableism.

Mit einem weiten, an Lemke (2010) angelehnten Begriff von Diskriminierung kommen verschiedene Ebenen in den Blick. Die Frage nach vorgeburtlicher Suche nach der Behinderung an Eltern von Kindern mit Behinderung kann als interaktionelle Diskriminierung von Eltern behinderter Kinder und ihren Kindern verstanden werden. Eine befürchtete Verschlechterung der gesellschaftlichen Stellung behinderter Menschen wegen PND ist zwar ein häufig genutztes, doch inhaltlich eher vages Argument, das den Diskriminierungsbegriff unzulässig ausdehnt. Auch das Argument der von behinderten Menschen gefühlten Diskriminierung bleibt unklar, zudem sind starke Rückkopplungseffekte der Debatte zu erwarten. Gesundheitsökonomische KostenNutzen-Rechnungen sind als organisationale Diskriminierung zu werten, sie stellen jedoch keinen legitimen Teil des Diskurses mehr dar. Alle diese Topoi funktionieren bei 
genauerer Betrachtung nicht ohne den Bezug zum Schwangerschaftsabbruch, können also zur Beantwortung der Frage, ob PND an sich diskriminierend wirke, nicht herangezogen werden.

Diskriminierung, die durch pränatale Diagnostik erfolgt, kann nur in Bezug auf das ableistische medizinische Modell von Behinderung beobachtet werden. Die Normalisierung der PND verstärkt das normative Verständnis von Behinderung als Defizit und von Behinderten als Leidende, zudem schränkt es die Handlungsoptionen schwangerer Frauen ein.

\section{Resümee}

Ist pränatale Diagnostik als Diskriminierung von Menschen mit Behinderung zu verstehen? Wie ich gezeigt habe, können die bisherigen Herleitungen einer Diskriminierung durch PND nicht überzeugen, genauso wenig jedoch Versuche ihrer Widerlegung. Das liegt zum einen daran, dass diese Frage meist gar nicht beantwortet wurde, sondern implizit die von der Diagnostik motivierten Schwangerschaftsabbrüche als Diskriminierungsgrund angesehen wurden. Auch die bisher vorgebrachten Argumente funktionieren, wenn überhaupt, nur in Bezug auf den Abbruch. Die Debatte darum, ob pränatale Diagnostik an sich als Diskriminierung verstanden werden kann, hat also eigentlich noch gar nicht angefangen.

Ich habe in diesem Beitrag möglicherweise mehr Fragen aufgeworfen, als ich beantwortet habe. Dies zeigt jedoch nur, dass in der Debatte bisher kaum valide Argumente ausgetauscht wurden. Mein Vorschlag ist daher mehrschichtig: Zum einen sollten die Debatten aus der Behindertenbewegung und den Disability Studies um das medizinische bzw. soziale Modell von Behinderung und den Ableism-Begriff aufgenommen werden. Zum anderen wäre in der Diskussion ein weiter und differenzierter Diskriminierungsbegriff zu nutzen, der strukturelle, nichtintentionale und diskursive Phänomene fassen kann. Zudem käme es dem Diskussionsniveau zugute, wenn transparenter gemacht würde, auf welche Sachverhalte man sich eigentlich jeweils bezieht, d.h. auf welche Art der pränatalen Diagnostik, und ob Schwangerschaftsabbrüche mitgedacht werden oder nicht.

Meine Anwendung des Modells der genetischen Diskriminierung von Lemke (2010) auf PND legt nahe, dass die Normalisierung der Verfahren, der defizitorientierte Blick, die sich verstärkende Angst vor Behinderung und die individualisierte Verantwortung der Schwangeren Aspekte der pränatalen Diagnostik sind, die als Diskriminierung begriffen werden können. Ob Diskriminierung der treffendste Begriff ist, um die problematische pränatale Suche nach Behinderungen kritisch zu beschreiben, soll damit nicht gesagt sein, ich denke aber plausibilisiert zu haben, dass es möglich 
ist, sie als solche zu verstehen. Dabei bleibt aber die Schwierigkeit bestehen, trennscharf zwischen den Effekten der PND und denen der Schwangerschaftsabbrüche zu unterscheiden.

Inwiefern ein solches weites Konzept von Diskriminierung für eine qualitative und quantitative Forschung operationalisierbar sein kann, stellt ein Desiderat dar. Angesichts der wenigen, mit geringen Teilnehmer*innenzahlen arbeitenden und größtenteils mittlerweile aufgrund gesetzlicher Änderungen veralteten Untersuchungen wäre es wünschenswert, dass zukünftige Studien mit einem komplexen Diskriminierungsbegriff arbeiten würden und so die Debatte um die durch pränatale Diagnostik geförderte Diskriminierung voranbringen könnten. Bisher gibt es im deutschsprachigen Raum noch kaum Untersuchungen dazu, wie behinderte Menschen über pränatale Diagnostik denken (Quaß, 2017). Diese müssten idealerweise so durchgeführt werden, dass dadurch auch der Topos, sich »diskriminiert zu fühlen «, reflektiert und besser verstehbar wird. Auch der Zusammenhang von Denormalisierungsangst, Ableism und pränataler Diagnostik ist noch zu wenig verstanden - besonders in Hinblick auf die Frage nach der Rolle der schwangeren Frau.

Die Klärung der Frage, ob und inwiefern PND als Diskriminierung zu fassen ist, erfordert aber auch eine Reflexion der gesellschaftlichen Macht- und Herrschaftsverhältnisse. Zu den sozialen Prozessen, die Ableism hervorbringen und verstärken, gehört auch das Angebot der pränatalen Diagnostik, das ein Defizitmodell von Behinderung plausibel erscheinen lässt.

\section{Anmerkungen}

1 Da es keine einheitliche Position der Betroffenen darüber gibt, wie Menschen mit Behinderung genannt werden möchten, werde ich - als Nichtbetroffene - die gängigen Selbstbezeichnungen »Menschen mit Behinderung« und »behinderte Menschen« verwenden. Zu Identitätskonzepten und Kategorienkritik in den Disablilty Studies vgl. Maskos (2019).

2 Die Kritik an pränatalen Untersuchungen und der normalisierten Suche nach Abweichungen im Rahmen der Schwangerschaftsvorsorge hat sich seit den 1980er Jahren vor allem im Feminismus entwickelt und umfasst neben den Diskriminierungsvorwürfen ein weites Feld an Debatten u.a. über eine Medikalisierung der Schwangerschaft, eugenische Entwicklungen, neoliberale Machbarkeitslogiken und einen individualisierten Selbstbestimmungsbegriff.

3 Die gesetzliche Regelung des Schwangerschaftsabbruchs ist in Deutschland kompliziert und widersprüchlich: Die embryopathische Indikation, die Abtreibungen wegen fötaler Behinderung legalisierte, wurde 1995 auf Druck von Behindertenverbänden und Kirchen wieder abgeschafft (Berg 2004, S. 44). Bei der Begründung wurde explizit Bezug auf das ein Jahr zuvor verabschiedete Diskriminierungsverbot für Menschen mit Behinderung (Artikel 3[3]) Bezug genommen. Ob das gesetzliche Diskriminierungsverbot für Föten gilt, wird in der Debatte allerdings eher bestritten (Birnbacher 2006, S. 326; Kösters 2014, S. 90ff.). Seitdem wird für Abbrüche nach pränataler Diagnose die medizinische Indikation angewendet, die eine Gefahr für das Leben oder eine 
schwerwiegende Beeinträchtigung des körperlichen oder seelischen Gesundheitszustandes der Schwangeren abwenden soll.

4 Diskriminierung wird vor allem als rechtlich zu lösendes Problem diskutiert, darauf soll in diesem Beitrag jedoch nicht weiter eingegangen werden.

5 Auch in den deutschsprachigen Disability Studies lässt sich kein ausgearbeiteter Diskriminierungsbegriff ausmachen.

6 In einer Befragung von Eltern von Kindern mit Trisomie 21 gaben 28,3\% der Mütter an, Vorwürfen von Verwandten, Freund*innen, Bekannten und ihren eigenen Eltern ausgesetzt gewesen zu sein, 72\% dieser Mütter und alle sich dazu äußernden Väter waren konkret gefragt worden, warum keine pränatale Diagnostik stattgefunden habe (vgl. unveröffentlichte Dissertation von Lümkemann, 2001, nach Lenhard et al., 2005, S. 35).

7 Diese Analyse sollte jedoch nicht dazu führen, alle Ängste umstandslos als Indikator für Diskriminierung und als relevant für politische Entscheidungen zu betrachten. Vielmehr sollten die Ängste auf eine Kopplung an tatsächlich von Diskriminierung betroffene Gruppen geprüft werden - ansonsten besteht die Gefahr, mit einem so ausgeweiteten Diskriminierungsbegriff die Ängste vor »Überfremdung«, »Christenverfolgung « oder »Frühsexualisierung « ernstnehmen zu müssen, statt sie als rechte Propaganda entlarven zu können (vgl. Sanders, Achtelik \& Jentsch, 2018, S. 79ff.).

8 Diese Überlegung ist auch sehr präsent in dem Buch der Journalistin Sandra Schulz (2017, S. 53) über ihre Schwangerschaft nach einer Trisomie 21-Feststellung.

\section{Literatur}

Achtelik, K. (2015). Selbstbestimmte Norm. Feminismus, Pränataldiagnostik, Abtreibung. Berlin: VerbrecherVerlag.

Achtelik, K. (2018/im Druck). Unsichtbare Geburten: Späte Schwangerschaftsabbrüche nach pränataler Diagnostik. In T. Jung (Hrsg.), Die Politik der Geburt. Kritische Perspektiven auf den Wandel von Gebären und Geburtshilfe. Bielefeld: transcript.

Baldus, M. (2006). Von der Diagnose zur Entscheidung: eine Analyse von Entscheidungsprozessen für das Austragen der Schwangerschaft nach der pränatalen Diagnose Down-Syndrom. Bad Heilbrunn: Klinkhardt.

Berg, U. (2004). Die Problematikder»eugenischen Indikation «als Rechtfertigungsgrund i. S. v. \$218allStGBn.F. http://geb.uni-giessen.de/geb/volltexte/2006/2753/pdf/BergUlrike-2004-11-18.pdf(08.04.2018).

Birnbacher, D. (2006). Selektion von Nachkommen. In D. Birnbacher, Bioethik zwischen Natur und Interesse (S. 315-336). Frankfurt am Main: Suhrkamp.

Boshammer, S. (2008). Diskriminierung. In S. Gosepath, W. Hinsch \& R. Celikates (Hrsg.), Handbuch der politischen Philosophie und Sozialphilosophie. Band 1 A-M (S. 232-238). Berlin: de Gruyter.

Degener, T. (2015). Vom medizinischen zum menschenrechtlichen Modell von Behinderung. Konzepte für Behindertenrecht und -politik. In I. Attia, S. Köbsell \& N. Prasad (Hrsg.), Dominanzkultur reloaded (S. 155-168). Bielefeld: transcript.

El-Mafaalani, A., Waleciak, J. \& Weitzel, G. (2017). Tatsächliche, messbare und subjektiv wahrgenommene Diskriminierung. In U. Hormel \& A. Scherr (Hrsg.), Diskriminierung: Grundlagen und Forschungsergebnisse (S. 173-189). Wiesbaden: VS.

Enquete-Kommission »Recht und Ethik der modernen Medizin« (2002). Schlussbericht. http://dip. bundestag.de/btd/14/090/140 90 20.pdf (08.04.2018).

Graumann, S. (2014). Die UN-Behindertenrechtskonvention und der Anspruch behinderter Menschen auf gesellschaftliche Anerkennung - sozialethische Überlegungen zur Praxis der Pränatal- und 
Präimplantationsdiagnostik. In G. Duttge, W. Engel \& B. Zoll (Hrsg.), »Behinderung« im Dialog zwischen Recht und Humangenetik (S. 71-82). Göttingen: Universitäts-Verlag.

Flaig, B. (2009). Forschungsprojekt Diskriminierung im Alltag: Wahrnehmung von Diskriminierung und Antidiskriminierungspolitik in unserer Gesellschaft. Baden-Baden: Nomos.

Füller, C. (2018). Gymnasium gegen Inklusion: Ungesundes Volksempfinden. Spiegel Online, 12.04.2018. www.spiegel.de/lebenundlernen/schule/bremen-schulleiterin-klagt-gegen-inklusion -ungesundes-volksempfinden-a-1202590.html (14.04.2018).

Hormel, U. \& Scherr, A. (2010). Einleitung: Diskriminierung als gesellschaftliches Phänomen. In U. Hormel \& A. Scherr (Hrsg.), Diskriminierung: Grundlagen und Forschungsergebnisse (S. 7-21). Wiesbaden: VS.

Jonas, K. (2014). Diskriminierung. In G. Endruweit, G. Trommsdorff \& N. Burzan (Hrsg.),Wörterbuch der Soziologie (S. 80-81). Konstanz: UVK.

Köbsell, S. (2015). Ableism. Neue Qualität oder salter Wein< in neuen Schläuchen? In I. Attia, S. Köbsell \& N. Prasad (Hrsg.), Dominanzkultur reloaded (S. 21-35). Bielefeld: transcript.

Kösters, A. (2014). Vorgeburtliche Selektion und Diskriminierung von Menschen mit Behinderungen: eine medizinethische Analyse. Münster: MV Wissenschaft.

Krones, T. (2008). Pränatal- und Präimplantationsdiagnostik: Diskriminierung von Menschen mit Behinderungen? In D. Gross, S. Müller \& J. Steinmetzer (Hrsg.), Normal - Anders - Krank? Akzeptanz, Stigmatisierung und Pathologisierung im Kontext der Medizin (S. 435-454). Berlin: Medizinisch Wissenschaftliche Verlagsgesellschaft.

Kuhlmann, A. (2011). Reproduktive Autonomie? Zur Denaturierung der menschlichen Fortpflanzung. In A. Kuhlmann, An den Grenzen unserer Lebensform: Texte zur Bioethik und Anthropologie (S. 85-107). Frankfurt am Main: Campus.

Lemke, T. (2006). Die Polizei der Gene. Formen und Felder genetischer Diskriminierung. Frankfurt am Main: Campus.

Lemke, T. (2010). Genetische Diskriminierung: Empirische Befunde und konzeptionelle Probleme. In U. Hormel \& A. Scherr (Hrsg.), Diskriminierung: Grundlagen und Forschungsergebnisse (S. 323-345). Wiesbaden: VS.

Lenhard, W., Henn, W., Ebert, H., Schindelhauer-Deutscher, J. \& Breitenbach, E. (2005). Januskopf der Diagnostik: Eltern von Kindern mit Behinderung im Spannungsfeld zwischen Unsicherheit und Ausgrenzung. Leben mit Down-Syndrom, 50, 30-37.

Lou, S., Petersen, O., Jørgensen, F., Lund, I., Kjaergaard, S. \& Vogel, I. (2018). National screening guidelines and developments in prenatal diagnoses and live births of Down syndrome in 1973-2016 in Denmark. Actaobstetricia et gynecologica Scandinavica, 97(2), 195-203.

Lübbe, W. (2003). Das Problem der Behindertenselektion bei der pränatalen Diagnostik und der Präimplantationsdiagnostik. Ethik in der Medizin, 15, 203-220.

Maskos, R. (2019/im Druck). Identität und Identitätspolitik: Welche Bedeutung haben sie für Menschen mit Behinderungen? In A. Waldschmidt (Hrsg.), Handbuch Disability Studies. Wiesbaden: Springer.

Parens, E. \& Asch, A. (2000). The disability rights. Critique of prenatal genetic testing: Reflections and recommendations. In E. Parens \& A. Asch (Hrsg.), Prenatal testing and disability rights (S. 3-44). Washington, D.C.: Georgetown University Press.

Quaß, M. (2017). Pränataldiagnostik und Schwangerschaftsabbruch aus Sicht von Menschen mit Trisomie 21. Unveröffentlichte Masterarbeit.

Renner, I. (2006). Schwangerschaftserleben und Pränataldiagnostik. Repräsentative Befragung Schwangerer zum Thema Pränataldiagnostik. Köln: BZgA.

Rommelspacher, B. (1999). Behindernde und Behinderte - politische, kulturelle und psychologische Aspekte der Behindertenfeindlichkeit. In B. Rommelspacher (Hrsg.), Behindertenfeindlichkeit: Ausgrenzungen und Vereinnahmungen (S. 7-37). Göttingen: Lamuv-Verlag. 
Sanders, E., Achtelik, K. \& Jentsch, U. (2018). Kulturkampf und Gewissen. Medizinethische Strategien der »Lebensschutz«-Bewegung. Berlin: Verbrecher-Verlag.

Scherr, A. (2017). Soziologische Diskriminierungsforschung. In A. Scherr, A. El-Mafaalani \& E. Yüksel (Hrsg.), Handbuch Diskriminierung (S. 39-58). Wiesbaden: Springer.

Tolmein, O. (Hrsg.). (1990). Geschätztes Leben: Die neue rEuthanasies-Debatte. Hamburg: Konkret-Verlag.

United Nations (2006). Übereinkommen über die Rechte von Menschen mit Behinderungen. http:// www.institut-fuer-menschenrechte.de/fileadmin/user_upload/PDF-Dateien/Pakte_ Konventionen/CRPD_behindertenrechtskonvention/crpd_b_de.pdf (08.04.2018).

Van den Daele, W. (2005). Vorgeburtliche Selektion: Ist die Pränataldiagnostik behindertenfeindlich? Leviathan-Sonderheft Biopolitik, 23, 97-123.

Volz, S. (2003). Diskriminierung von Menschen mit Behinderung im Kontext von Präimplantations- und Pränataldiagnostik. In S. Graumann \& K. Grüber (Hrsg.), Medizin, Ethik und Behinderung (S. 72-89). Frankfurt am Main: Mabuse-Verlag.

Waldschmidt, A. (2009). »Normalität« und »Behinderung« im Alltagswissen: Diskursanalyse eines Internetforums. SWS-Rundschau,49, 314-336.

Weingart, P., Kroll, J. \& Bayertz, K. (1992). Rasse, Blut und Gene: Geschichte der Eugenik und Rassenhygiene in Deutschland. Frankfurt am Main: Suhrkamp.

\section{Die Autorin}

Kirsten Achtelik ist Promovendin an der Humboldt-Universität zu Berlin, freie Journalistin und Autorin. Ihre Arbeitsschwerpunkte sind: Feministische Theorien und Bewegungen, Ableism, Biopolitik und Reproduktionstechnologien.

Kontakt: www.kirsten-achtelik.net 